\title{
Letter to the editor re Sredni et al.: spontaneous involution of pediatric low-grade gliomas: high expression of cannabinoid receptor 1 (CNR1) at the time of diagnosis may indicate involvement of the endocannabinoid system (2016)
}

\author{
Paul Steinbok ${ }^{1}$ (1)
}

Received: 20 December 2016/Accepted: 21 December 2016/Published online: 28 December 2016

(C) Springer-Verlag Berlin Heidelberg 2016

\section{Dear Editor:}

I wish to compliment Sredni et al. [1] for their report "Spontaneous involution of pediatric low-grade gliomas: high expression of cannabinoid receptor 1 (CNR1) at the time of diagnosis may indicate involvement of the endocannabinoid system." They found that incompletely resected pediatric low-grade gliomas which remained stable or involuted spontaneously expressed higher levels of the cannabinoid receptor 1 (CNR1) than those tumors which progressed. They suggested that spontaneous involution of these pediatric low-grade gliomas may have been induced by endocannabinoids. I found this particularly interesting in the light of our previous report of two incompletely resected septum pellucidum pediatric low-grade astrocytomas that involuted spontaneously concurrent with the use of cannabis [2]. I wonder if one might be able to identify a population of low-grade gliomas and perhaps other brain tumors with elevated levels of CNR1 that might be particularly susceptible to involution with the use of cannabis. This "drug" could then be potentially used as a chemotherapeutic agent in such patients.

Compliance with ethical standards

Conflict of interest The author states that there is no conflict of interest.

\section{References}

1. Sredni ST, Huang CC, Suzuki M, Pundy T, Chou P, Tomita T (2016) Spontaneous involution of pediatric low-grade gliomas: high expression of cannabinoid receptor 1 (CNR1) at the time of diagnosis may indicate involvement of the endocannabinoid system. Child's Nervous System : ChNS: Official Journal of the International Society for Pediatric Neurosurgery 32:2061-2067

2. Foroughi M, Hendson G, Sargent MA, Steinbok P (2011) Spontaneous regression of septum pellucidum/forniceal pilocytic astrocytomas - possible role of cannabis inhalation. Child's Nervous System: ChNS: Official Journal of the International Society for Pediatric Neurosurgery 27:671-679
Paul Steinbok

psteinbok@cw.bc.ca

Division of Neurosurgery, BC Children's Hospital and University of British Columbia, 4480 Oak St., Room K3-159, Vancouver, BC V6h 3V4, Canada 